\title{
Self-grafting carbon nanotubes on polymers for stretchable electronics ${ }^{\star}$
}

\author{
Piero Morales ${ }^{1,2}$, Slavianka Moyanova ${ }^{3}$, Luigi Pavone ${ }^{3}$, Laura Fazi ${ }^{2,4}$, Daniele Mirabile Gattia ${ }^{1}$, Bruno Rapone ${ }^{1}$, \\ Anderson Gaglione ${ }^{3}$, and Roberto Senesi ${ }^{2,4,5, a}$ \\ 1 ENEA Centro Ricerche della Casaccia, S. Maria di Galeria, 00123 Roma, Italy \\ 2 Centro NAST, Università degli Studi di Roma "Tor Vergata", 00133 Roma, Italy \\ 3 IRCCS Neuromed, 86077, Pozzilli, Italy \\ 4 Dipartimento di Fisica, Università degli Studi di Roma "Tor Vergata", 00133 Roma, Italy \\ ${ }^{5}$ Centro Fermi-Museo Storico della Fisica e Centro Studi e Ricerche "Enrico Fermi", 00184 Roma, Italy
}

Received: 12 January 2018 / Revised: 30 March 2018

Published online: 6 June 2018

(C) Società Italiana di Fisica / Springer-Verlag GmbH Germany, part of Springer Nature, 2018

\begin{abstract}
Elementary bidimensional circuitry made of single-wall carbon-nanotube-based conductors, selfgrafted on different polymer films, is accomplished in an attempt to develop a simple technology for flexible and stretchable electronic devices. Unlike in other studies of polymer-carbon nanotube composites, no chemical functionalization of single-wall carbon nanotubes is necessary for stable grafting onto several polymeric surfaces, suggesting viable and cheap fabrication technologies for stretchable microdevices. Electrical characterization of both unstretched and strongly stretched conductors is provided, while an insight on the mechanisms of strong adhesion to the polymer is obtained by scanning electron microscopy of the surface composite. As a first example of technological application, the electrical functionality of a carbonnanotube-based 6-sensor (electrode) grid was demonstrated by recording of subdural electrocorticograms in freely moving rats over approximately three months. The results are very promising and may serve as a basis for future work targeting clinical applications.
\end{abstract}

\section{Introduction}

Since the discovery of carbon nanotubes (CNT) and of their interesting mechanical and electrical properties [1], large efforts have been devoted to obtaining composite materials based on CNT, both in their single-wall (SWCNT) and multi-wall form (MWCNT) [2]. As is widely recognized, due to their inert graphitic structure, carbon nanotubes are non-reactive and hardly soluble both in water and in organic solvents, which limits the possibilities of producing composites and, in spite of effort, no general recipe has been found [3-5]. Limited dispersion in liquids is obtained by the use of surfactants, e.g., sodium-dodecyl-benzene sulphonate (SDBS) and, only to some lesser degree, CNT can be suspended in alcohol and other organic solvents. DNA or other long-chain polymers, such as sodium-carboxy methyl cellulose, can wrap around CNT and can thus make the complex soluble in water. In addition, damaging the graphite structure of CNT by strong acidic attack produces a number of electron devoid sites, making them partly soluble and available also for functionalization with hydrophilic groups. However, such damage deteriorates both the mechanical and the electrical performance of CNT, so that at most some tradeoff can be found which may be convenient for specific applications. Furthermore, CNT of all types, being many orders of magnitude longer than their diameter, invariably twist and bundle together, making unextricable randomly coiled bundles of different forms, which, while further limiting the possibility of uniform functionalization, have, on the other hand, interesting elastic properties $[6$ 9]. In spite of these difficulties, many achievements of polymer-CNT composites have been obtained and successfully tested $[2,10-16]$.

\footnotetext{
* Supplementary material in the form of a .pdf file available from the Journal web page at https://doi.org/10.1140/epjp/i2018-12040-0

a e-mail: roberto.senesi@uniroma2.it
} 
The process of grafting of CNT onto polymer film surfaces is of particular importance for obtaining flexible and stretchable planar devices $[8,17]$. Indeed, such surface composites would find wide applications for sensors, electronic devices, artificial muscles, implantable prosthetic aids [18-25], extensible displays and even smart luminous clothing. Growth, grafting by chemical modification or plasma treatment, deposition, extrusion and lamination of MWCNT in polymeric films have been explored, but relatively less attention has been paid to the electrical properties of surfacegrafted conductors based on SWCNT. The present work is devoted to the investigation of the electrical conductivity of bundled SWCNT wires self-grafted on the surface of polymeric films with different elastic/plastic behaviour. We thus exploit the natural rearrangement of polymeric flexible chains around the much more rigid and yet very thin SWCNTs $(0.7 \mathrm{~nm}$ diameter $)$; these can penetrate more or less deeply the bulk of the polymer, so that intertwining with its chains becomes frequent enough to produce a grafting force that depends on the type of polymer. Beyond the wide issue of stretchable electrical connection on a $\mathrm{mm} / \mathrm{cm}$ scale, and more generally of "stretchy" electronics, we also explored, as a practical application of these compliant and moldable surface composites, the possibility of recording electrocorticogram (ECoG) in freely behaving laboratory rats; this is obtained with a SWCNT grid immersed in a biocompatible poly-ethylene (PE) plastic film membrane placed subdurally on the cortical surface of the rats (between the dura and the pia mater). The use of this type of flat, stretchable CNT-based probe is justified by previous findings [26-30] showing that neurons easily colonize CNT bundles, and by the need of fabrication of fully biocompatibile, elastic and plastic neuroprosthetic devices for Brain Machine Interface (BMI) applications. Furthermore, metal-free conductors could represent a strong asset for NMR tomographies in biomedical applications, while the perspective of using ink-jet printing of carbon nanotubes is particularly appealing for low-cost mass production of stretchable devices. Also, the inert nature of graphitic carbon, and its immersion in biocompatible polymers, is a further advantage in medical and prosthetic devices.

\section{Materials and experimental section}

\subsection{Materials}

Purified SWCNT produced by the HiPCo method [31] were purchased from Carbon Nanotechnologies Inc.. Impurities declared by the manufacturer are less than $5 \%$, mainly consisting of excess metal clusters catalizing CNT initial growth. As is widely known, this material appears as a black fluffy powder made of intertwined clusters of dimensions ranging from fractions of a millimeter to few $\mu \mathrm{m}$. Their average density is very low, so that these clusters easily float in air, while they tend to stick to surfaces where they deposit, making a precise measurement of the overall mass rather difficult. On Scanning Electron Microscopy (SEM) analysis, the material shows the complex coiling of single nanotubes, as well as of their bundles. The diameter of the individual SWCNT in this material is $0.7 \mathrm{~nm}$ as declared by the manufacturer, which is approximately 40 times smaller than that of multi-walled carbon nanotubes (MWCNT, Aldrich), having an external diameter of the order of 20-40 nm. Both types of CNT samples have mixed chirality. Representative images of the single- and multi-walled carbon nanotubes that we used, and of their very different arrangements in clusters, are given in the Supplementary Material (SM) fig. S1.

In our experiments aiming at grafting individual conducting tracks of CNT onto a polymeric surface, we chose thin films of a number of polymers having different elastic and plastic properties: 1) medium-density poly-ethylene (MD$\mathrm{PE}) ; 2$ ) high-density poly-ethylene (HD-PE); 3) acrylonitrile/butadiene (nitrile), a very elastic polymer; 4) poly-vinyl chloride (PVC); 5) Parafilm M, a very plastic film made of paraffin waxes mixed with poly-olefins, that was found to be ideal for neuron cells growth [32]; 6) natural rubber latex.

\subsection{Conductive tracks fabrication}

A suspension of partly shortened SWCNT was obtained by pouring $10 \mathrm{mg}$ of powder into $10 \mathrm{ml}$ distilled $\mathrm{H}_{2} \mathrm{O} /$ ethanol in the ratio 70/30 and adding $30 \mathrm{mg}$ sodium-dodecyl-benzene sulphonate (SDBS). The mixture was quickly sonicated for 10 minutes and then ball milled for 2 minutes in a Spex 8000 ball miller with a stainless steel jar. The suspension obtained in this way was not at all uniform but finer than before ball milling, and it was possible to pass it through a $200 \mu \mathrm{m}$ needle using a $10 \mathrm{ml}$ syringe for casting on the substrate.

The polymeric film substrate was very slightly stretched on the outer wall of a bulk aluminium cylindrical drum ( $4 \mathrm{~cm}$ diameter), perpendicularly to its axis, and fixed by adhesive tape at its ends. A stencil was made of 4 parallel steel wires $(0.5 \mathrm{~mm}$ diameter) in two couples spaced $1 \mathrm{~cm}$ from each other, with adjustable spacing between wires of each couple (generally $0.5 \mathrm{~mm}$ for one couple and $0.8 \mathrm{~mm}$ for the other); such stencil was also stretched on the drum over the polymer, so that the wires were forced to be parallel and to adhere perfectly to the film substrate slightly squeezing it against the aluminium drum surface. The drum had an electric heater at the center to adjust the temperature of the polymer film. This was measured by a Chromel-Alumel $0.5 \mathrm{~mm}$ diameter stainless steel armoured thermocouple, inserted in a $0.6 \mathrm{~mm}$ diameter bore just below the drum wall surface. While the temperature was kept at approximately $80^{\circ} \mathrm{C}$, the CNT suspension was slowly cast through the needle between the $1 \mathrm{~mm}$ spaced wires of 
each of the two couples. The solvent evaporated quickly at that temperature, leaving a brown/black deposit on the polymer surface. The operation was repeated until the space between the steel wires was completely filled and appeared uniformly black. Each CNT track was then lightly brushed to remove non-adhering flakes and further cleaned by a jet of purified air; then we raised the temperature to approximately 1-2 degrees below the polymer melting temperature (e.g., $115^{\circ} \mathrm{C}$ for our MD-PE film, $250^{\circ} \mathrm{C}$ for PVC) and maintained for a few minutes, then the drum was cooled again, the stencil was carefully removed and the specimen was examined. The first examination was made by an optical stereomicroscope at 50× magnification, then the film was observed by SEM after a light gold evaporation.

This technique is simpler, quicker and more economic than other methods necessary for large area uniform CNT coatings [33].

\subsection{Mechanical characterization}

We first performed the commonly used sticky tape stripping test for a qualitative assessment of the stability of adsorbed layers on flat surfaces. This was made for SWCNT layers self-grafted on our polymer film substrates, and a variety of results ranging from extremely good and stable to weak adhesion was obtained. Details are reported in the SM, fig. S3. A stress-strain characterization was also necessary since grafting of the SWCNT layer on the polymer surface may affect significantly its mechanical properties. This was performed using an analytical balance, by hooking one end of the nanotube coated polymer film stripes to a $120 \mathrm{~g}$ mass on the balance plate, and lifting the other end by controlled extensions while reading the weight decrease measured by the balance scale.

\subsection{Electrical characterization}

We carried out the first electrical characterization of the conducting CNT/polymer film wires by measuring their conductance as a function of track length. This was performed by platinum wire microprobes micrometrically shifted by a micrometric slide (Carl Suss) along a $0.8 \mathrm{~mm}$ wide track on a MD-PE film glued on a rigid substrate. Further characterization was made by measuring the current through the conducting CNT tracks versus applied voltage and versus film stretching. To this purpose the film was immobilized on a micrometric slide, with one side gripped onto the static part and the other gripped to the cursor part. Particular care was taken to avoid sliding of the film out of the copper gripping plates as a consequence of film stretching and thinning. This was obtained by inserting thin strips of sanding tissue on both sides of the gripping plates (see fig. S7 of the SM), making sure that the CNT tracks were in firm contact with the copper. We placed the slide under the objective of a stereomicroscope and observed the process of stretching of the CNT tracks deposited on the polymer. Simultaneously, resistance values of CNT tracks were acquired by an Agilent 3458A digital multimeter.

\subsection{Preparation of a six-sensor device for in vivo ECoG in rats}

Due to the need of an easily 3D moldable device made of a biocompatible material, the sensor grid was made on an MD-PE film substrate (thickness $25 \mu \mathrm{m}$ and dimensions $5.5 \times 5.5 \mathrm{~mm}$ ). The polymer film of 40 mm diameter was slightly stretched across a circular metal frame, which was fixed on an $x, y, z$ micrometric slide. The perfectly flat framed film was precisely positioned in the focal point of a $50 \mathrm{~mm}$ focal length lens, on which the beam from the $2 \mathrm{nd}$ harmonic of a Q-switched Nd-Yag laser $(532 \mathrm{~nm})$ was shone perpendicularly to the film surface; six micro apertures were then micro-drilled in it by pulsed laser ablation. The laser intensity was kept just above the threshold of plasma formation as a compromise for quick drilling and a reasonably round shape of the apertures with diameter of approximately 50-60 $\mu \mathrm{m}$. Fine adjustment of the beam intensity was obtained by suitably delaying the Q-switch pulse with respect to the flash lamp trigger. The position of the six apertures was determined with $10 \mu \mathrm{m}$ precision, according to the predetermined design, by shifting the micrometric slide in the $x, y$ directions with respect to the laser beam focal point. Then we deposited six $0.8 \mathrm{~mm}$ wide SWCNT tracks in straight paths over the drilled microapertures and over a set of seven parallel metal wires, which connected the apertures to the output conductive pads. The round micrometric areas of the CNT conductive tracks exposed by the micro-drilled apertures served as sensing electrodes, while the polymer film substrate provided the necessary insulating membrane between electrodes and between wires and brain tissue. Further details and images of the complete device are given in the SM. Then, by means of silver paint droplets, we soldered the SWCNT wires to copper wires serving as an intermediate connection to an output connector. This was followed by drying and covering the connection with epoxy resin, to make it stronger and more reliable. Further details and images of the complete device are given in the SM.

The six-electrode grid was placed subdurally on the rat's cortical surface by appropriate surgery and operative treatments following the authorized rules for using animals in chronic experiments. These experiments were conducted in accordance with the guidelines approved by the institutional committee of I.R.C.S.S. Neuromed Institute (Pozzilli, Italy), where this part of the study was carried out. We followed the protocols for surgery commonly used for subdural implantation of flat electrode grids on the cortical surface of rodents [34]. Then, we recorded ECoG signals from each electrode of the grid starting one week after implantation. 


\section{Results}

It should first be observed that grafting a CNT layer of thickness in the range of 10-20 $\mu$ m involves two very different types of interaction: the first is the adhesion of carbon nanotubes to the polymer surface, the second is the adhesion of the upper layers of CNTs onto the polymer grafted nanotubes, which is mainly due to the random entanglement of coiled and twisted nanotubes. We found that the first type of interaction is much stronger than nanotube-nanotube entanglement. The deposited SWCNT layer that forms the base of the conductive track was surprisingly stable and firmly attached to the polymer surface, in spite of the lack of any sort of chemical treatment or covalent bonding. This was true for most of the explored polymers, as demonstrated by the sticky tape test, although with variable adhesion: the best results in terms of adhesion were obtained with MD-PE, PVC and natural rubber latex film substrates, while the weakest adhesion was observed for nitrile films; on the other hand, this test was not possible for Parafilm M, which is too soft a material to whitstand the sticky tape stripping. For polyethylenes, rubber latex and PVC, we even attempted to crush the polymer film or to mechanically scratch the tracks and this never removed the surface grafted layer, although damage to the upper layers, and a consequent increase in the resistance, occurred. We believe that such good adhesion is due to both buckling of the polymer chains onto the carbon nanotube bundles [35, 36] and to the SWCNT diameter which allows penetration into the polymer structure [37]. The polymer chain stiffness, the history of the polymer casting and the chirality and buckling of the SWCNT bundles to each other seem to be also important for this good adhesion [37,38]. In general, consecutive tape strippings led to a roughly constant wear of the SWCNT thick layer, while the thin adhering layer remained strongly grafted onto the polymer. Stabilization of the upper layers of the thick carbon nanotube films is, in any case, obtained by soaking it with a PE/Xilene solution, leading to devices that are fully resistant to 10 consecutive tape strippings, as is shown in detail in fig. S3 of the SM.

The resistance of the self-grafted SWCNT wires clearly decreases with increasing thickness and is of the order of 2-3 kOhm/cm for an approximately $10 \mu \mathrm{m}$ thick, $500 \mu \mathrm{m}$ wide, track. This is low enough to allow for the majority of possible applications. Even after complete removal of the CNT upper layers, resistance remains in the hundreds of $\mathrm{kOhm} / \mathrm{cm}$ range. The strongly non-linear behaviour of the resistance versus strain plots in the high strain region would be particularly useful for high-sensitivity strain sensors.

Figure 1 shows SEM images of portions of the bundled SWCNT layer grafted on a MD-PE film after attempting to mechanically detach (delaminate) it from the polymer. We can see that in spite of the lifting up of the SWCNT layer edge from the polymer, it still holds onto the surface by means of many residual nanotube cables anchoring the deposit in its place.

Figure 2 shows optical and SEM microscope images of similar conductive tracks as patterned on the polymer film, after removal of the masking structures, and on severe (>100\%, figs. 2(a), (b), (c)) and mild (10\%) stretching (fig. 2(e)) along the direction of the wires. When the polymer is severely stretched, cracks develop in the upper layers of the conductive tracks as observed by optical microscopy and SEM characterization (figs. 2(a), (b), (c)). Even for large strains, of the order of 120-150\%, such cracks do not completely cross the wire width, so that a bellows-like structure is formed. The electrical conductivity is preserved although with increased resistance, as we shall see in the following; furthermore, the narrower gaps that develop are always bridged by SWCNT (fig. 2(c)). In agreement with what reported by Liu et al. [22], an increase of the strain range that maintains high conductance can be obtained by casting the SWCNT suspension on pre-stretched polymer films.

Optical microscopy also showed that the SWCNT tracks were more rigid than the polymer film: indeed some wrinkles formed around the wire due to its grafting (fig. 2(d)). These disappeared on slightly stretching the polymeric substrate, while leaving the tracks firmly anchored to the polymer surface (fig. 2(e)). The limits in lateral precision, due to the SWCNT clusters size, are also visible in fig. 2(e). Removal of the stencil was a frequent cause for irregular wire edge since SWCNT clusters often strongly adhere even to metal masks.

Figure 3 depicts stress-strain curves (squares and full circles) for $31.5 \mathrm{~mm}$ long, $3 \mathrm{~mm}$ wide and $25 \mu \mathrm{m}$ thick MD-PE stripes, uncoated and coated by a self-grafted 10-15 $\mu$ m thick SWCNT layer.

We can clearly see that the effect of the SWCNT-grafted layer was to substantially increase the material stiffness at small strains until the stretched nanotube bundles were forced to disentangle, which produced a clear double slope behaviour of the stress-strain curve.

Figure 3 investigates also the correlation between strain and increase of electrical resistance of the same above mentioned wire. The resistance versus strain plot (stars) shows again a double slope behaviour, but the change of slope occurs at higher strains compared to the stress-strain plots. We may interpret this observation as follows: the initial disentanglement of CNT bundles when stretched beyond their elastic limit decreases the film stiffness even if bundles remain in contact with each other; only further stretching of the film causes a disconnection of the CNT bundles and subsequent steeper increase in the resistance.

A simple Ohmic behaviour was obtained, as expected, on measuring the resistance of several polymer surface grafted SWCNT wires as a function both of the distance between measuring points along the wires, and of the voltage applied to the SWCNT wire. Details are reported in fig. S6 of the SM. 

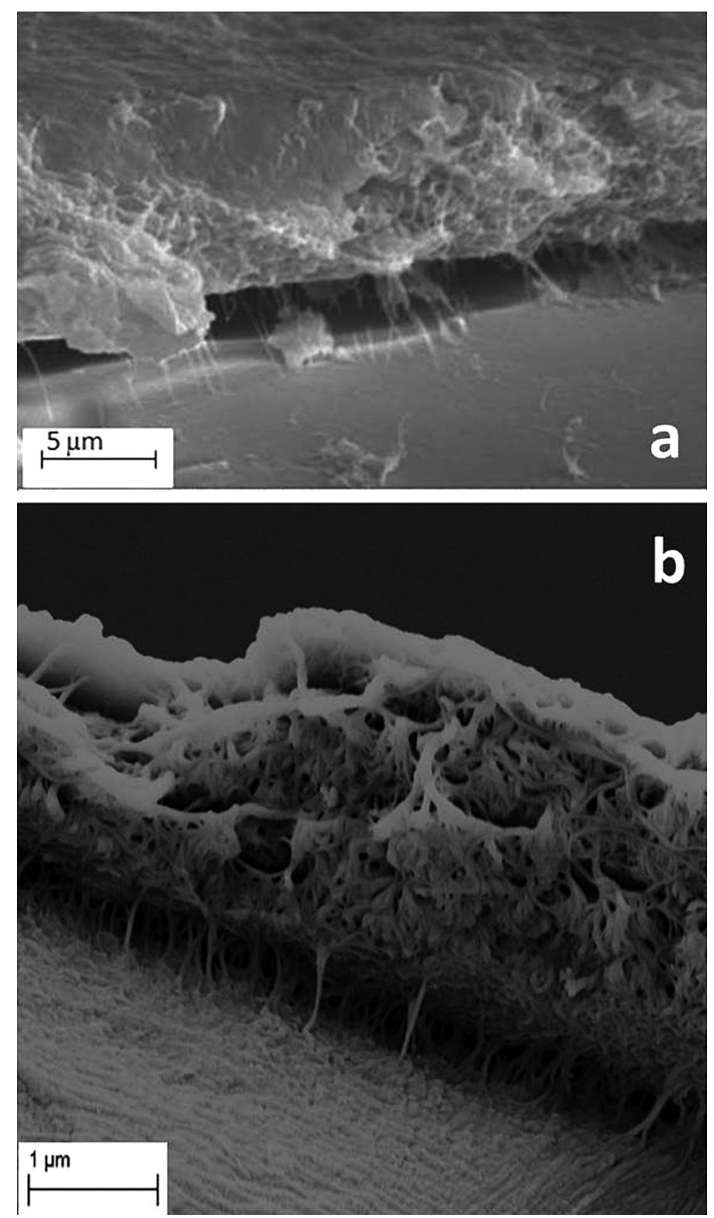

Fig. 1. SEM images of SWCNT layers on MD-PE at different angles show many nanotube bundles firmly bonding the conductive layer to the polymer surface. Panel (a) shows strained nanotubes on attempting detachment of the SWCNT layer from the polymer (note that some nanotubes are broken due to high tension). Panel (b) is taken, on a different specimen, at a very low angle, nearly parallel to the surface at the edge of the SWCNT layer and shows the large amount of binding nanotubes well rooted into the polymer. Both images were recorded after a few sticky tape strippings.

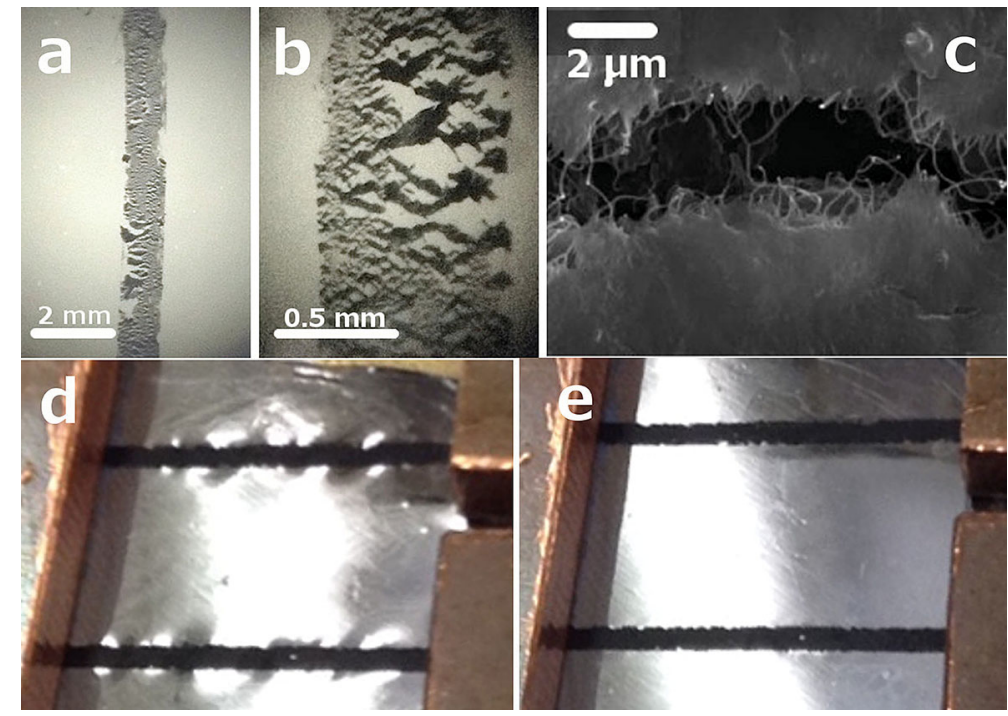

Fig. 2. Characterization of stretched SWCNT wire grafted on MD-PE. Panels (a) and (b): Severe stretching. Doubling the length of the polymer substrate produces a bellows like structure, as shown in (a), (b), that is still conductive. Panel (c): The SEM image also shows single SWCNT bundles bridging the majority of the cracks that form the extended bellows. Panel (d): The unstretched, suspended polymer film shows wrinkling due to SWCNT grafting, which disappears on slight stretching (e). 


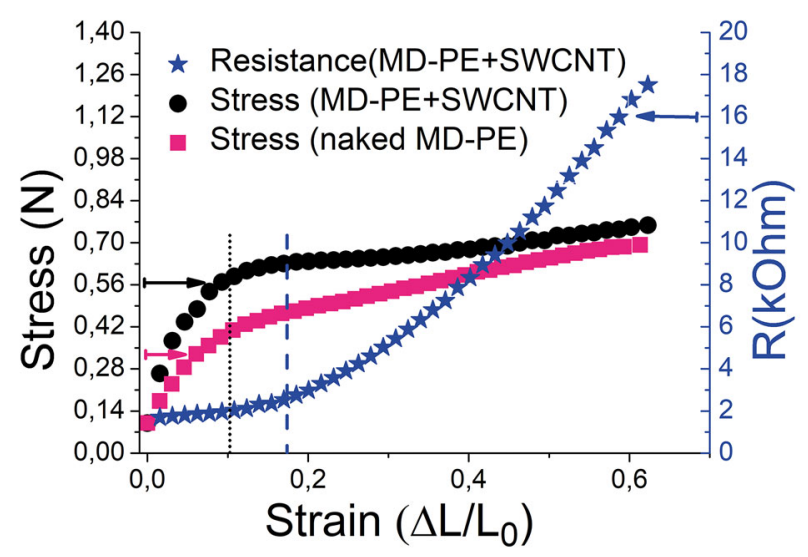

Fig. 3. Stress-strain plots for a naked (squares) and SWCNT-coated (full circles) MD-PE strips (left scale) and resistance-strain plot (stars) simultaneously recorded on the same specimen. Vertical dotted lines mark the change of slopes. The stripe is $3 \mathrm{~mm}$ wide, $31.5 \mathrm{~mm}$ long and $25 \mu \mathrm{m}$ thick, while SWCNT coating is $10-15 \mu \mathrm{m}$ thick.

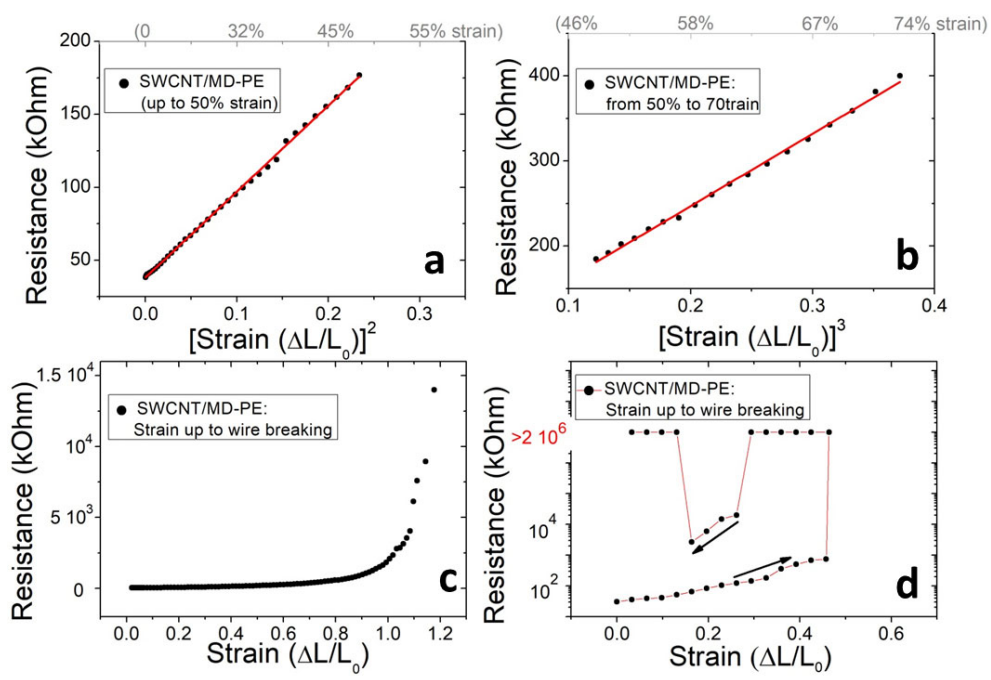

Fig. 4. Resistance versus strain plots for a $1 \mathrm{~mm}$ wide, $14.7 \mathrm{~mm}$ long and $15-20 \mu \mathrm{m}$ thick SWCNT wire self-grafted on MD-PE for different relative strain regions: (a) 0-50\% with a linear fit of the data versus $\left[\Delta L / L_{0}\right]^{2}$; (b) $50 \%-100 \%$ with a linear fit of the data versus $\left[\Delta L / L_{0}\right]^{3}$. In both (a) and (b) the upper scale reports for convenience the corresponding relative strains. (c) $0-120 \%$. Thickness of the SWCNT layer is approximately 15-20 $\mu \mathrm{m}$. (d) Breakdown threshold for a $10 \mu \mathrm{m}$ thick SWCNT/MD-PE wire is below $50 \%$ strain. Note that on decreasing the strain after breakdown some random conduction region can be found.

When the polymer was stretched in the direction of the wire, the resistance of a $15 \mu \mathrm{m}$ thick, $800 \mu \mathrm{m}$ wide film grafted wire, increased non-linearly with its extension $\Delta L$. Figure 4 shows the measurements of resistance versus relative strain $\left(\Delta L / L_{0}\right)$ for strains up to approximately $50 \%$ (fig. $4\left(\right.$ a), plotted against $\left.\left(\Delta L / L_{0}\right)^{2}\right)$, from $50 \%$ to $70 \%$ (fig. 4(b), plotted against $\left.\left(\Delta L / L_{0}\right)^{3}\right)$, and in the whole range up to $120 \%$ plotted against $\Delta L / L_{0}$ (fig. $4(\mathrm{c})$ ). Observing that for small strains (up to approximately 30\%) the width $w$ of the CNT track remains constant, the observed quadratic increase in fig. 4(a) can be compatible with a simple Ohmic behavior if we suppose that the SWCNT film thickness decreases linearly with strain:

$$
R=\rho \cdot \frac{L}{\sigma}=\rho \cdot \frac{L}{t \cdot w}
$$

with $\rho=$ resistivity; $\sigma=\operatorname{section} ; L=$ length; $t=$ film thickness; $w=$ film width.

On supposing $\Delta t=k / \Delta L$, where $k$ has the dimension of an area and the width $w$ is constant (the film is stretched in the direction of the wire length),

$$
\Delta R=\frac{(r \cdot \Delta L)}{\left[\left(\frac{k}{\Delta L}\right) \cdot w\right]} \rightarrow \Delta R=k^{\prime} \Delta L^{2},
$$

where $k^{\prime}=r / k w$ has the dimension of a volume resistivity. 


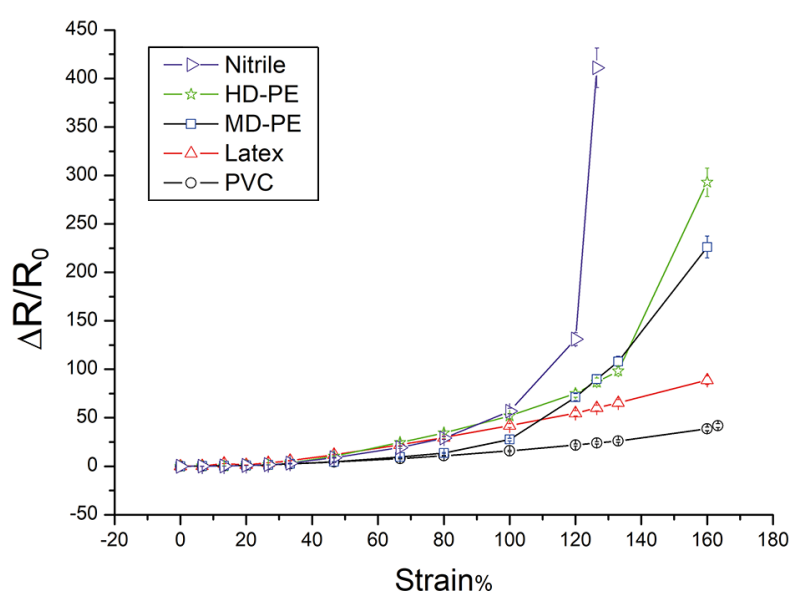

Fig. 5. Increase of resistance versus strain for a $6 \mathrm{~mm}$ wide, 15-20 $\mu \mathrm{m}$ thick SWCNT track on different polymeric film substrates.

This simplified description only accounts for the low strain behaviour where the CNT layer can be considered roughly uniform. Indeed, the assumption $\Delta t=k / \Delta L$ is probably true on average, although fig. 2 shows that almost empty cracks progressively develop in a finely distributed but discrete way.

Stretching the SWCNT/MD-PE composite film beyond $50 \%$ yields higher power dependencies on strain (figs. 4(b), (c)) due to progressive loss of internal connectivity of the wire; and above $120 \%$ an unpredictable failure of the conductor was observed. Reducing the thickness and width of the wire lowers the breakdown threshold, which was $35-50 \%$ for a $500 \mu \mathrm{m}$ wide and approximately $10 \mu \mathrm{m}$ thick wire (fig. $4(\mathrm{~d})$ ). On releasing the strain after breakdown some regions of re-established conductor continuity could be found, but the wire was permanently ruined after breakdown in all specimens. On the other hand, releasing the strain before reaching the failure threshold extension, the resistance of the wire decreases and, depending on the material elasticity, it approaches the original values in a more or less extended time, so that some hysteresis is observed; in most cases, however, it never reaches the resistance values relative to the original, unstretched polymer film. The hysteresis is clearly related to the slow and incomplete recovery of the original extension of the polymeric film. Stretch-release curves for different substrates are reported in fig. S8 of the SM.

A comparison of the reliability of SWCNT wires grafted on different polymer films was made by measuring the resistance of wires of similar thickness and width as a function of the applied strain. Figure 5 reports such comparison. We can see that the best overall behaviour is obtained with the PVC substrate, where the resistance increase is minimal, followed by rubber latex, MD-PE, HD-PE and finally nitrile where the wire approches full breaking for strains above 100\%. Parafilm M grafted CNT wires whitstand only extremely limited strains due to strongly non-uniform plastic deformation of the substrate, causing high straining of the CNT bundles in very limited regions, and sharp cracks in the wire; because of their unreliable behaviour, data for Parafilm $\mathrm{M}$ are not reported in the figures. We also note that at low strains MD-PE behaves better than rubber latex, and similarly to PVC, while its resistance has a strong increase for strains higher than $100 \%$. This could be due to exceeding the onset of the MD-PE plastic behaviour. For the particular application of Electrocorticogram recording in living laboratory animals, the MD-PE substrate was thus chosen, since only limited stretching of the device is required, together with a good plastic behaviour and full biocompatibility.

We also tested the reliability of these self-grafted wires by repeated stretch-release cycles. Figure 6 shows the wire resistance for 100 cycles of stretch-release at different amounts of stretching, from 10\% to 100\% of the original wire length. Resistance increases with increasing strain as already shown, but it remains stable after the first 20 cycles, showing that the SWCNT/polymer composite is indeed a conductor with very reliable electrical properties under mechanical loads.

Since most of the studies available in the literature have been performed using MWCNT rather than SWCNT, we also compared a SWCNT wire grafted on PE with a MWCNT wire made in exactly the same way on the same film close to each other. Contrary to the behaviour of SWCNT/polymer film tracks, MWCNT tracks were much less stable. The delamination from the polymeric substrate already observed by others [39] occurred after inducing any type of deformation of the MWCNT film, leaving only a very non-uniform distribution of small nanotube clusters (figs. 7(a)(c)). These remaining clusters had very limited conductivity and behaved differently from the SWCNT tracks, as shown in fig. 7(d) for strains up to $50 \%$. Resistance is an approximate factor $>10^{3}$ higher, as expected for such thin and non-uniform wire; also, the behavior is non-Ohmic at low strains, strongly deviating from what we observed for the SWCNT wire. This behaviour may be interpreted as a lower penetration of the 40 times wider MWCNT into the polymer, together with a more difficult rearrangement of the polymeric chains around such larger and stiffer carbon structure as predicted by simulation studies $[35,36]$. Furthermore, due to their coarse grained structuring on a micrometer scale (see SEM characterization in the on-line SM), MWCNT offer a much lower contact surface to the polymer film, compared to the more uniform SWCNT distribution. 


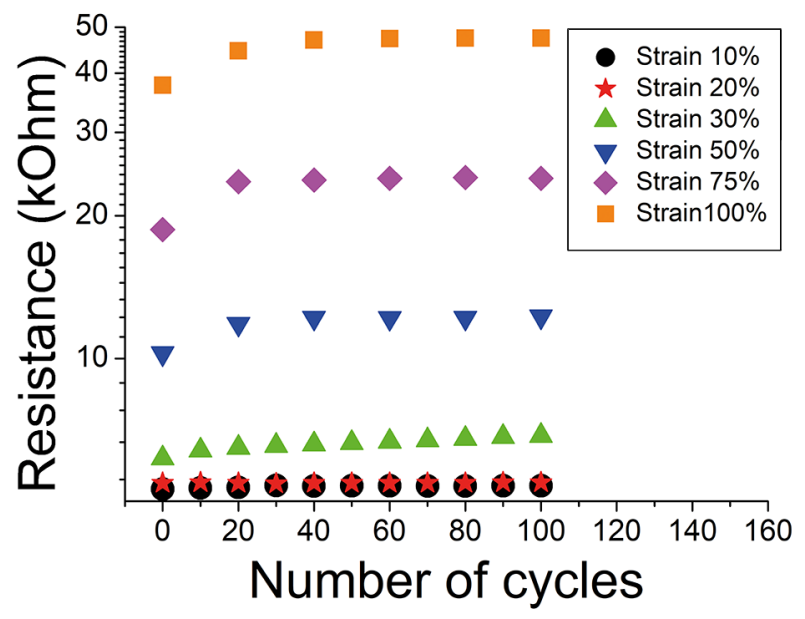

Fig. 6. Resistance of a $3 \mathrm{~mm}$ wide MD-PE stripe coated with SWCNT versus number of stretch-release cycles for different strains.
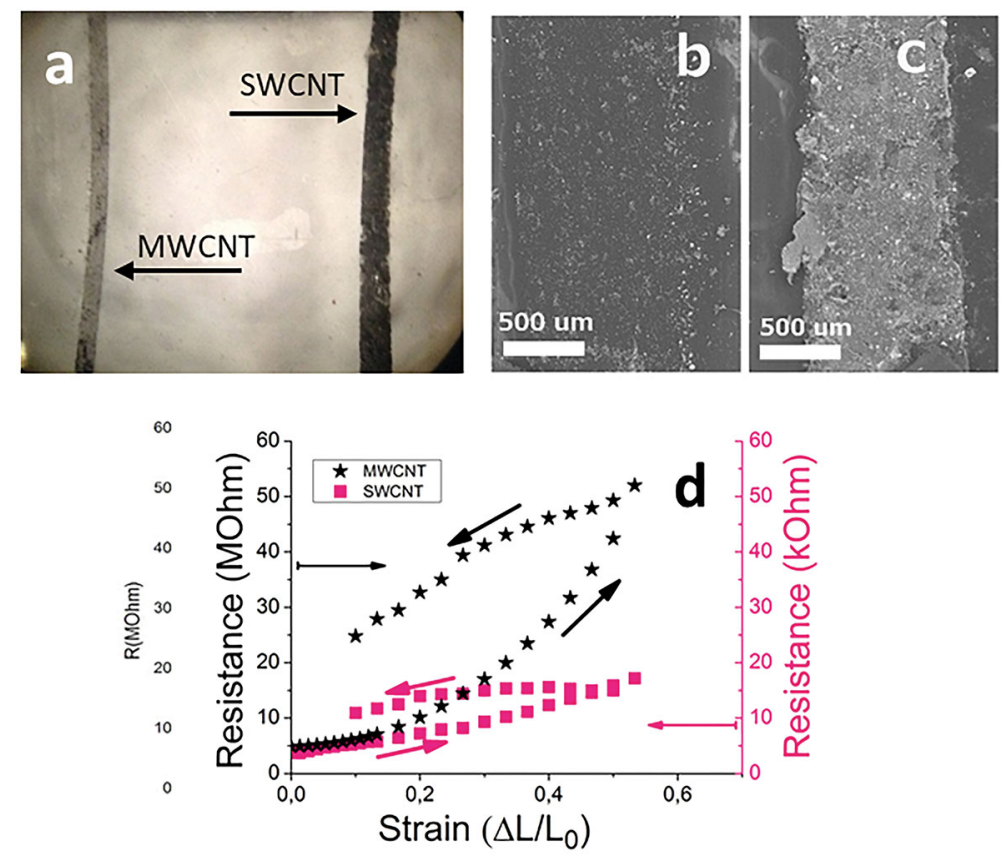

Fig. 7. (a) Optical microscopy shows the widely different grafting of MWCNT in form of a wire on MD-PE (left) compared to that of SWCNT (right) on the same substrate. (b) and (c): SEM imaging of a MWCNT and of a SWCNT track, respectively. (d) Comparison of resistance versus strain behaviour for a SWCNT (squares, scale on the right) and a MWCNT (stars, scale on the left) wire on the same MD-PE film. Length of the wires is $15 \mathrm{~mm}$. Note that the scale for MWCNT is in MOhm, while the scale for the SWCNT wire is $\mathrm{kOhm}$.

\section{Recording of rat electrocorticogram in vivo}

One of our future goals is to use this manufacturing process to create SWCNT-based electrode grids to target clinical applications, such as control of epilepsy in drug-resistant patients and help paralyzed patients affected by stroke by enhancing and improving communication and control by means of brain-computer interfacing. We present here a short description of our first results concerning the reliability of a SWCNT/MD-PE-based 6-electrode grid by recording ECoG activity in a freely moving laboratory rat. We placed the grid subdurally to record the ECoG directly from the surface of the cerebral cortex because it is well known that scalp, dura mater and intermediate tissues strongly attenuate and distort brain signals [40]. The ECoG represents a class of semi-invasive, i.e. intracranial but not intracortical, recording technique and compared with EEG, it offers higher quality as control signal for BMI applications because of its higher spatial resolution, improved signal-to-noise ratio and lower contaminations with movement artefacts [41,42]. 


\section{a}

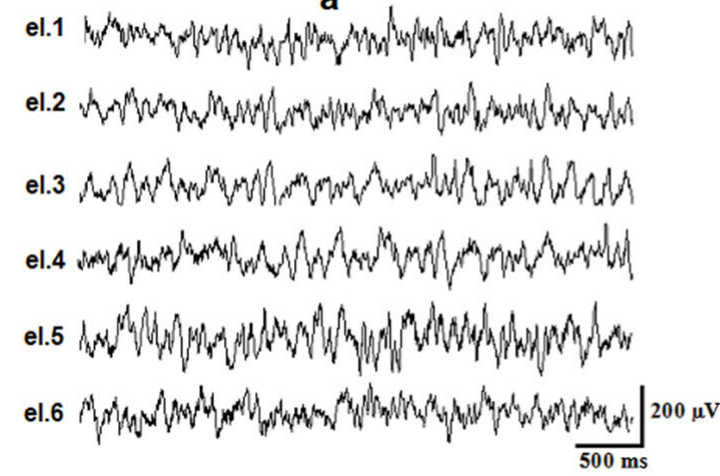

b

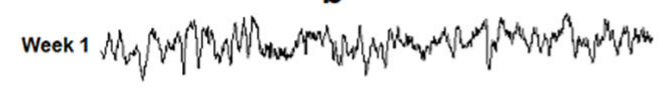

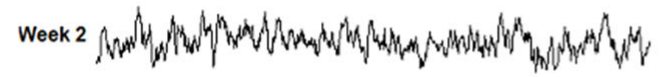

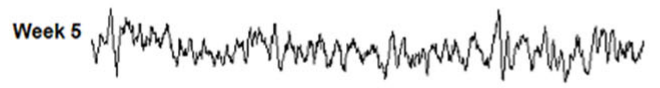

Week 11 /
C
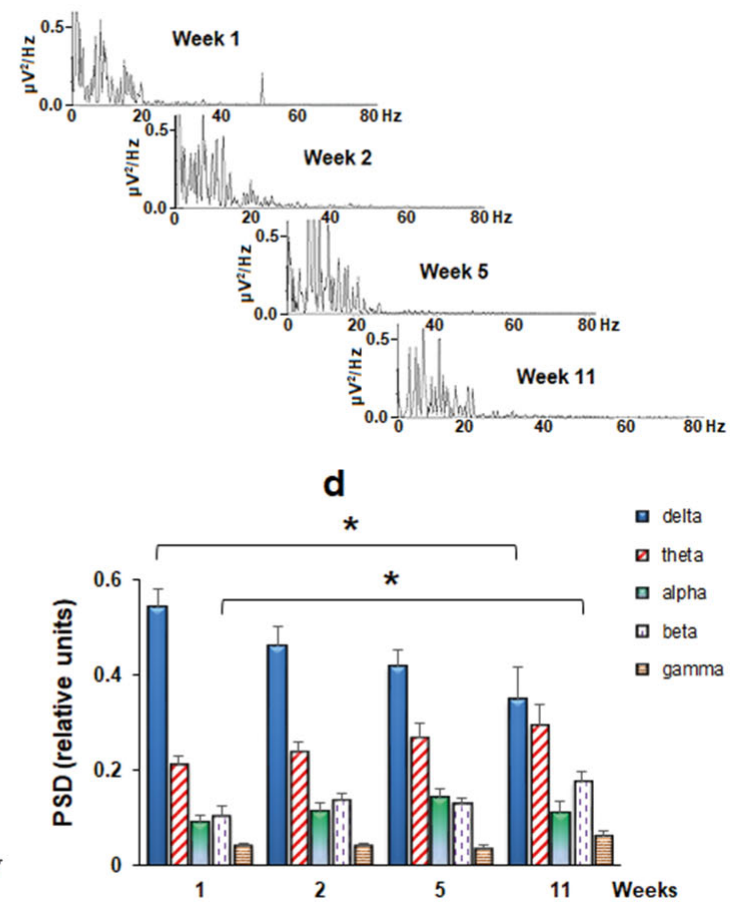

Fig. 8. The ECoG recorded by means of SWCNT/MD-PE grid subdurally implanted in a laboratory rat. (a) Representative monopolar $4 \mathrm{~s}$ ECoGs recorded in a free-behaving video-monitored rat in a state of quiet waking from all 6 electrodes of the SWCNT/MD-PE grid implanted subdurally 11 weeks earlier. (b) Representative monopolar $4 \mathrm{~s}$ ECoGs from one of the six electrodes at 1, 2, 5, and 11 weeks. All electrodes captured reliable ECoG beginning from the second week after the implantation and until the end of the experiment (week 11). (c) Power spectral densities (PSDs) of the $4 \mathrm{~s}$ ECoG epochs, which are shown on (b). (d) Histogram plots of PSD values (mean + standard error of mean) obtained by integration of normalized PSD for delta, theta, alpha, beta, and gamma frequency bands for ten $4 \mathrm{~s}$ epochs in ECoG recordings at week $1,2,5$, and $11 .{ }^{*}$ The significance level was set at 0.05 (Bonferroni test).

As is generally accepted, impedance, number of channels detecting electrical activity, amplitude, and frequency of the signals are the benchmarks for ECoG quality, as well as the viability of the nearby tissue. In order to verify whether the electrical characteristics of the electrodes are sufficient to operate in biological frequency bands and ascertain the electrodes stability over time, we performed impedance measurements (from the first day) and ECoG recordings (from the first week) once a week over 11 weeks after the implantation of the grid. We observed some increase of the impedance only the first week after the implantation, while later-on the impedances of all 6 electrodes of the grid were much lower (see SM). The initial increase in the impedance is a typical behaviour of implanted microelectrodes and grids, most probably due to the tissue response [34,43]. Having low impedance of the electrode/tissue interface is very important to maintain signal quality during recording. In fact, we recorded reliable ECoG signal starting from the second week until the end of the experiment with $100 \%$ of functioning electrodes (figs. 8(a), (b)). We carried out spectral analysis of the ECoG using power spectral density (PSD) application of the AcqKnowledge 4.1.1 software (BIOPAC Syst, Inc, U.S.A.). The PSDs were calculated with a Hamming-windowed 1600-point fast Fourier transform using standard Welch procedure $(0.195 \mathrm{~Hz}$ resolution). The analysis showed that the PSDs (fig. 8(c)) of $4 \mathrm{~s}$ ECoG epochs (fig. 8(b)) were typical for the functional state of passive waking, when the rat is quiet, does not move or sleep [44]. In accordance with the high level of impedance measured the first week following the surgery (see the SM), the ECoG during that week was not very stable with slow deviations and appearance of line $50 \mathrm{~Hz}$ noise (figs. 8(b), (c)) due probably to not yet good contact of the electrodes with the underlying cortical tissue $[34,43]$. We tested the electrical stability of the longitudinal ECoG monitoring by means of ANOVA with repeated factor "Weeks" on normalized values of PSDs (against the total power over the entire frequency range) of 10 ECoG $4 \mathrm{~s}$ epochs in the standard five frequency bands: delta $(0.5-4 \mathrm{~Hz})$, theta $(5-8 \mathrm{~Hz})$, alpha $(9-12 \mathrm{~Hz})$, beta $(13-30 \mathrm{~Hz})$, and gamma $(31-70 \mathrm{~Hz})$. The ANOVA and post-hoc Bonferroni test rejected the hypothesis that the power-frequency characteristics of ECoG will change over time in our chronic experimental setting from week 2 until week 11 after the grid implantation, which indicated to stability of ECoG over approximately three months. The variations in the power distribution were due mainly to difference between near times (week 1) versus far times (week 11) (fig. 8(d)). The decrease in delta and increase in beta at week 11 relative to week 1 might be due to the stabilization of the signal at later time points. The analysis of 
video records made simultaneously with the ECoG showed that the rat displayed normal behaviour without any signs of pathology. In our experiments, we did not compare the recorded ECoG with signals recorded by means of other grids, which incorporate metal disks (e.g., platinum-iridium alloy, gold, etc.) routinely used in humans [45] or any other grids fabricated using other methods/materials. Generally, grids with metal electrodes are not very suitable for prolonged chronic applications because they are heavy and undergo oxidation and degradation of characteristics over time [46]. There is a bulk of study demonstrating the superiorities in electrical and biocompatibility characteristics of CNT electrodes over other types of electrodes, e.g., titanium-nitride electrodes [47].

We will continue our efforts to follow requirements for biocompatibility that will allow for chronic implantation of such devices over more prolonged periods and to improve spatial and frequency resolution of the cortical recording, which means to fabricate SWCNT-based ECoG grids with smaller interelectrode distances, bigger number of sensors, and use of acquisition setup to catch faster ECoG activity. We believe that the SWCNT/MD-PE grids described in this study may be used in future for control of motor disability in humans after stroke $[41,45,48]$ and seizures in patients with intractable epilepsy [49].

\section{Conclusions}

We have demonstrated reliable self-grafting of SWCNT bundles on several polymer films, as compared to the more commonly used MWCNT, which normally need some type of chemical or physical functionalization in order to bind polymers. Such good adhesion may be related to the higher degree of penetration of SWCNT into the polymers, due to their smaller diameter compared to MWCNT. This leads to the availability of easy and cheap fabrication of stretchable conductors, which may be more or less elastic or plastic, depending on the polymeric film substrate. Demonstrating such surface grafting of conducting and elastic carbon structures is important in the perspective of industrial fabrication of complex stretchable circuitry or sensing devices by drop casting and printing methodologies. The composite surface material has interesting mechanical properties, adding considerable strength to the polymeric films for small strains. For larger strains, where the stretched carbon nanotube bundles disentangle, the dependence of resistance on strain is similar to the stress-strain dependence of the naked film. Although we cannot be sure about the mechanisms of self-grafting, the SEM characterization showed that, unlike the much larger MWCNT, the SWCNT, probably because of their small size, penetrated into the polymer structure, where they remained buried and entangled via buckling mechanisms. Variable rigidity of the polymer backbone may account for different adhesion properties of different polymeric substrates. Once grafted to polyethylene substrate films, the SWCNT layers of the order of few microns were very stable and robust, which allowed us to fabricate a six-electrode flat stretchable grid and implant it subdurally on the cortical surface of the brain of a free-moving laboratory rat. This device was safe during approximately three months of testing with no apparent damage to the animal health and effective in capturing reliable and stable ECoG activity. Within the increasing investigation on stretchable conductors based on carbon nanotubes/polymer composites, our findings, due to the easy and inexpensive methodology, appear particularly promising for immediate and broad ranging application.

This work was partially supported within the Project SMART Campus (Regione Lazio 2015-2017), within the Framework agreement (Accordo Quadro) between IRCCS NEUROMED and Università degli Studi di Roma "Tor Vergata" (2014).

\section{Author contribution statement}

The manuscript was written through contributions of all authors. All authors have given approval to the final version of the manuscript. PM and LP contributed equally.

\section{References}

1. S. Ijima, Nature 354, 56 (1991).

2. N. Tsubokawa, Polym. J. 37, 637 (2005).

3. L. Vaisman, H.D. Wagner, G. Marom, Adv. Colloid Interface Sci. 128-130, 37 (2006).

4. M.J. Rahman, T. Mieno, J. Nanomater. 2014, 508192 (2014).

5. D. Yang, X. Zhang, C. Wang, Y. Tang, J. Li, J. Hu, Prog. Nat. Sci. 19, 991 (2009).

6. R.S. Ruoff, D. Qian, W.K. Liu, C. R. Phys. 4, 993 (2003).

7. P. Salvetat, A.J. Kulik, J.M. Bonard, G.A.D. Briggs, T. Stockli, K. Metenier, S. Bonnamy, F. Beguin, N.A. Burnham, L. Forro, Adv. Mater. 11, 161 (1999).

8. C.Q. Ru, Phys. Rev. B 62, 10405 (2000). 
9. D.A. Walters, L.M. Ericson, M.J. Casavant, J. Liu, D.T. Colbert, K.A. Smith, R.E. Smalley, Appl. Phys. Lett. 74, 3803 (1999).

10. Q. Cao, S.H. Hur, Z.T. Zhu, Y. Sun, C. Wang, M.A. Meitl, M. Shim, J.A. Rogers, Adv. Mater. 18, 304 (2006).

11. D. Baskaran, J.W. Mays, M.S. Bratcher, Angew. Chem. Int. Ed. Engl. 43, 2138 (2004).

12. N. Satyanarayana, K. Rajan, S. Sinha, L. Shen, Tribol. Lett. 27, 181 (2007).

13. J.H. Shi, B.X. Yang, S.H. Goh, Eur. Polym. J. 45, 1002 (2009).

14. K. Zhang, J.Y. Lim, H.J. Choi, Diamond Relat. Mater. 18, 316 (2009).

15. X.L. Wu, P. Liu, eXPRESS Polym. Lett. 4, 723 (2010).

16. M.T. Byrne, Y.K. Gun'ko, Adv. Mater. 22, 1672 (2010).

17. T. Sekitani, Y. Noguchi, K. Hata, T. Fukushima, T. Aida, T. Someya, Science 321, 1468 (2008).

18. M.K. Shin, J. Oh, M. Lima, M.E. Kozlov, S.J. Kim, R.H. Baughman, Adv. Mater. 22, 2663 (2010).

19. Z. Zhan, R. Lin, V.T. Tran, J. An, Y. Wei, H. Du, T. Tran, W. Lu, ACS Appl. Mater. Interfaces 9, 37921 (2017).

20. F. Vitale, S.R. Summerson, B. Aazhang, C. Kemere, M. Pasquali, ACS Nano 9, 4465 (2015).

21. X. Song, S. Liu, Z. Gan, L. Qiang, H. Cao, H. Yan, Microelectron. Eng. 86, 2330 (2009).

22. Z.F. Liu, S. Fang, F.A. Moura, J.N. Ding, N. Jiang, J. Di, M. Zhang, X. Lepró, D.S. Galvão, C.S. Haines, N.Y. Yuan, S.G. Yin, D.W. Lee, R. Wang, H.Y. Wang, W. Lv, C. Dong, R.C. Zhang, M.J. Chen, Q. Yin, Y.T. Chong, R. Zhang, X. Wang, M.D. Lima, R. Ovalle-Robles, D. Qian, H. Lu, R.H. Baughman, Science 349, 400 (2015).

23. T.Y. Tsai, C.Y. Lee, N.H. Tai, W.H. Tuan, Appl. Phys. Lett. 95, 013107 (2009).

24. I.R. Minev, P. Musienko, A. Hirsch, Q. Barraud, N. Wenger, E.M. Moraud, J. Gandar, M. Capogrosso, T. Milekovic, L. Asboth, R.F. Torres, N. Vachicouras, Q. Liu, N. Pavlova, S. Duis, A. Larmagnac, J. Vörös, S. Micera, Z. Suo, G. Courtine, S.P. Lacour, Science 347, 6218 (2015).

25. Y.C. Chen, H.L. Hsu, Y.T. Lee, H.C. Su, S.J. Yen, C.H. Chen, W.L. Hsu, T.R. Yew, S.R. Yeh, D.J. Yao, Y.C. Chang, H. Chen, J. Neural Eng. 8, 034001 (2011).

26. G. Gabriel, R. Gomez, M. Bongard, N. Benito, E. Fernandes, R. Villa, Biosens. Bioelectron. 24, 1942 (2009).

27. A. Shoval, C. Adams, M. David-Pur, M. Shein, Y. Hanein, Front. Neuroeng. 2, 4 (2009).

28. M. David-Pur, L. Bareket-Keren, G. Beit-Yaakov, D. Raz-Prag, Y. Hanein, Biomed. Microdev. 16, 43 (2014).

29. C.M. Lin, Y.T. Lee, S.R. Yeh, W.L. Fang, Biosens. Bioelectron. 24, 2791 (2009).

30. M. Shein, A. Greenbaum, T. Gabay, R. Sorkin, M. David-Pur, E. Ben-Jacob, Y. Hanein, Biomed. Microdev. 11, 495 (2009).

31. M.J. Bronikowski, P.A. Willis, D.T. Colbert, K.A. Smith, R.E. Smalley, J. Vac. Sci. Technol. A 19, 1800 (2001).

32. S.J. Yoo, Y. Nam, J. Neurosci. Methods 204, 28 (2012).

33. R.C. Tenent, T.M. Barnes, J.D. Bergeson, A.J. Ferguson, B. To, L.M. Gedvilas, M.J. Heben, J.L.J. Blackburn, Adv. Mater. 21, 3210 (2009).

34. C. Henle, M. Raab, J.G. Cordeiro, S. Doostkam, Biomed. Microdev. 13, 59 (2011).

35. S.S. Tallury, M.A. Pasquinelli, J. Phys. Chem. B 114, 4122 (2010).

36. S.S. Tallury, M.A. Pasquinelli, J. Phys. Chem. B 114, 9349 (2010).

37. C.Y. Wei, NanoLett. 6, 1627 (2006).

38. C. Ke, M. Zheng, I.T. Bae, G. Zhou, J. Appl. Phys. 107, 104305 (2010).

39. A.D. Willey, Thin Films of Carbon Nanotubes and Nanotube/Polymer Composites, Thesis, Brigham Young University (2012).

40. G. Buzsaki, C.A. Anastassiou, Ch. Koch, Nat. Rev. Neurosci. 13, 407 (2012).

41. E.C. Leuthardt, G. Schalk, J.R. Wolpaw, J.G. Ojemann, D.W. Moran, J. Neural Eng. 1, 63 (2004).

42. G. Schalk, K.J. Miller, N.R. Anderson, J.A. Wilson, M.D. Smyth, J.G. Ojemann, D.W. Moran, J.R. Wolpaw, E.C. Leuthardt, J. Neural Eng. 5, 75 (2008).

43. J.C. Williams, J.A. Hippensteel, J. Dilgen, W. Shain, D.R. Kipke, J. Neural Eng. 4, 410 (2007).

44. K.J. Maloney, E.G. Cape, J. Gotman, B.E. Jones, Neuroscience 76, 541 (1997).

45. J.C. Sanchez, A. Gunduz, P.R. Carney, J.C. Principe, J. Neurosci. Methods 167, 63 (2008).

46. S.F. Cogan, Annu. Rev. Biomed. Eng. 10, 275 (2008).

47. F. Sauter-Starace, O. Bibari, F. Berger, P. Caillat, A.L. Benabid, in 4th International IEEE/EMBS Conference on Neural Engineering, Antalya (2009) pp. 112-115.

48. K.J. Wisneski, N. Anderson, G. Schalk, M. Smyth, D. Moran, E.C. Leuthardt, Stroke 39, 3351 (2008).

49. J. Yuan, Y. Chen, E. Hirsh, Neurol. Sci. 33, 723 (2012). 